\title{
Parity Violation in the Scattering of a Proton by Carbon and Oxygen
}

\author{
A. I. Milstein ${ }^{a, b}$, N. N. Nikolaev ${ }^{c, ~ *}$, and S. G. Salnikov ${ }^{a, b}$ \\ ${ }^{a}$ Budker Institute of Nuclear Physics, Siberian Branch, Russian Academy of Sciences, Novosibirsk, 630090 Russia \\ ${ }^{b}$ Novosibirsk State University, Novosibirsk, 630090 Russia \\ ${ }^{c}$ Landau Institute for Theoretical Physics, Russian Academy of Sciences, Chernogolovka, Moscow region, 142432 Russia \\ *e-mail: nikolaev@itp.ac.ru \\ Received November 3, 2021; revised November 3, 2021; accepted November 3, 2021
}

\begin{abstract}
The effects of parity violation in the interaction of relativistic polarized protons with ${ }^{12} \mathrm{C}$ and ${ }^{16} \mathrm{O}$ nuclei are discussed. Within the Glauber approach, estimates are obtained for $P$-odd asymmetries in the total and elastic scattering cross sections, the dissociation cross section, and in the inelastic scattering cross section with meson production. Our calculations show that asymmetry should be most noticeable in the elastic cross section and in the dissociation cross section.
\end{abstract}

DOI: $10.1134 / \mathrm{S} 0021364021220033$

\section{INTRODUCTION}

At present, the effects of $P$-parity violation in the scattering of leptons by nucleons at not very high energies have been studied in detail both theoretically and experimentally. The obtained experimental data are well described within the Standard Model. There is much less experimental information on parity violation in nucleon-nucleon scattering, in nucleonnucleus scattering, and in nucleus-nucleus scattering [1-7]. The theoretical predictions for these processes are very different [8-18]. Polarization experiments at the NICA collider $[19,20]$ can make an important contribution to understanding the phenomenon of parity violation in the nucleon sector. Possible experiments at NICA to search for parity violation in the interaction of longitudinally polarized protons or deuterons with an unpolarized target were discussed in $[21,22]$. Estimates of the $P$-odd asymmetry in nucleon-nucleon scattering at NICA energies are given in [23], and in proton-deuteron scattering in [24].

The total nucleon-nucleon scattering cross section is the sum of the elastic scattering cross section and the cross section for inelastic scattering accompanied by the production of mesons. Quasi-elastic scattering accompanied by the emission of nucleons or the excitation of nuclei also contributes to the total cross section for nucleon-nucleus and nucleus-nucleus scattering. Since the effects of parity violation are small, when planning experiments, it is necessary to find processes in which these effects are enhanced.

From an experimental point of view, dense nuclear targets, such as carbon or water [4], are convenient. Therefore, it is of undoubted interest to study the effects of $P$-parity violation in the scattering of polarized protons by nuclei from the point of view of the possible enhancement of the $P$-odd effect by the number of nucleons in the nucleus. In this work, we investigate the $P$-parity violation in the scattering of a polarized proton by the ${ }^{12} \mathrm{C}$ and ${ }^{16} \mathrm{O}$ nuclei.

\section{WEAK INTERACTION EFFECTS IN PROTON-NUCLEUS SCATTERING}

In our work, we use the Glauber approach [25-27]. In this approach, in a frame where both particles are relativistic, the amplitude $T$ of elastic scattering of a proton by the ${ }^{12} \mathrm{C}$ and ${ }^{16} \mathrm{O}$ nuclei has the form $(\hbar=c=1)$

$$
\begin{gathered}
T\left(\mathbf{q}_{\perp}\right)=-2 i \int d^{2} \rho e^{-i \mathbf{q} \perp \cdot \mathbf{\rho}}\left[1-e^{-\mathscr{T}(\mathbf{\rho})}\right], \\
\mathscr{T}(\mathbf{\rho})=\frac{i}{2} \int \frac{d^{2} Q_{\perp}}{(2 \pi)^{2}} \\
\times e^{i \mathbf{Q}_{\perp} \cdot \boldsymbol{\rho}} S\left(\mathbf{Q}_{\perp}\right)\left[T^{p p}\left(\mathbf{Q}_{\perp}\right)+T^{p n}\left(\mathbf{Q}_{\perp}\right)\right] .
\end{gathered}
$$

Here, $T^{p p}\left(\mathbf{Q}_{\perp}\right)$ and $T^{p n}\left(\mathbf{Q}_{\perp}\right)$ are proton-proton and proton-neutron scattering amplitudes, respectively, and $S\left(\mathbf{Q}_{\perp}\right)$ is a form factor, which in the shell model equals

$$
S\left(\mathbf{Q}_{\perp}\right)=\left[Z-\frac{(Z-2)}{6} Q_{\perp}^{2} a^{2}\right] \exp \left(-\frac{1}{4} Q_{\perp}^{2} a^{2}\right),
$$

where $Z$ is the charge of the corresponding nucleus. For carbon we used the value $a=1.6 \mathrm{fm}$, and for oxygen $a=1.7 \mathrm{fm}$. The elastic cross section $\sigma_{\mathrm{el}}$ and the 
total cross section $\sigma_{\text {tot }}$ for the scattering of a proton by a nucleus are given by the formulas

$$
\begin{gathered}
\sigma_{\mathrm{el}}=\int \frac{d^{2} q}{(4 \pi)^{2}}\left|T\left(\mathbf{q}_{\perp}\right)\right|^{2} \\
=2 \int d^{2} \rho\left[1-\operatorname{Re} e^{-\mathscr{T}(\boldsymbol{\rho})}-\frac{1}{2}\left(1-e^{-2 \operatorname{Re} \mathscr{T}(\boldsymbol{\rho})}\right)\right], \\
\sigma_{\text {tot }}=-\operatorname{Im} T(0)=2 \int d^{2} \rho\left[1-\operatorname{Re} e^{-\mathscr{T}(\boldsymbol{\rho})}\right] .
\end{gathered}
$$

The cross section $\sigma_{\text {dis }}$ for dissociation with excitation or emission of nucleons from the nucleus and the cross section $\sigma_{\text {inel }}$ with production of mesons have the forms

$$
\begin{gathered}
\sigma_{\mathrm{dis}}=\int d^{2} \rho\left[e^{-2 \operatorname{Re} \mathcal{T}(\boldsymbol{\rho})}\left(e^{\Omega(\boldsymbol{\rho})}-1\right)\right], \\
\sigma_{\text {inel }}=\int d^{2} \rho\left[1-e^{-2 \operatorname{Re} \mathcal{T}(\boldsymbol{\rho})} e^{\Omega(\boldsymbol{\rho})}\right], \\
\Omega(\boldsymbol{\rho})=\frac{1}{4} \iint \frac{d^{2} Q_{\perp}}{(2 \pi)^{2}} \frac{d^{2} Q_{\perp}^{\prime}}{(2 \pi)^{2}} e^{i\left(\mathbf{Q}_{\perp}-\mathbf{Q}_{\perp}^{\prime}\right) \cdot \boldsymbol{\rho}} S\left(\mathbf{Q}_{\perp}-\mathbf{Q}_{\perp}^{\prime}\right) \\
\times\left[T^{p p}\left(\mathbf{Q}_{\perp}\right) T^{p p *}\left(\mathbf{Q}_{\perp}^{\prime}\right)+T^{p n}\left(\mathbf{Q}_{\perp}\right) T^{p n *}\left(\mathbf{Q}_{\perp}^{\prime}\right)\right] .
\end{gathered}
$$

Note that $\sigma_{\text {tot }}=\sigma_{\mathrm{el}}+\sigma_{\mathrm{dis}}+\sigma_{\text {inel }}$.

We represent the amplitudes $T^{p p}\left(\mathbf{q}_{\perp}\right)$ and $T^{p n}\left(\mathbf{q}_{\perp}\right)$ as the sum of the contributions of the strong and weak interactions, $T^{p N}\left(\mathbf{q}_{\perp}\right)=T_{s}^{p N}\left(\mathbf{q}_{\perp}\right)+T_{W}^{p N}\left(\mathbf{q}_{\perp}\right)$, where $N=p, n$. At NICA energies, the following parametrization can be used for the contribution of strong interaction [28]:

$$
\begin{gathered}
T_{s}^{p N}\left(\mathbf{q}_{\perp}\right)=-\delta_{\lambda_{1} \lambda_{3}} \delta_{\lambda_{2} \lambda_{4}}(\epsilon+i) \sigma_{s} \exp \left(-\frac{1}{2} B q_{\perp}^{2}\right), \\
\epsilon=-0.5, \quad \sigma_{s}=50 \mathrm{mb}, \quad B=9 \mathrm{GeV}^{-2},
\end{gathered}
$$

where $\lambda_{1}$ and $\lambda_{2}$ are the helicities of the initial particles and $\lambda_{3}$ and $\lambda_{4}$ are the corresponding helicities of the final particles $\left(\lambda_{i}= \pm 1\right)$. Using this parametrization, we find the contribution of strong interaction to the cross sections for proton-nucleus scattering:

$$
\begin{gathered}
\mathscr{T}_{s}(\boldsymbol{\rho})=(1-i \epsilon) \Phi(\rho), \quad \Omega_{s}(\boldsymbol{\rho})=\gamma \Phi(\rho), \\
\Phi(\rho)=\frac{\sigma_{s}}{\pi R^{2}}\left[Z-\frac{2 a^{2}(Z-2)}{3 R^{2}}\left(1-\frac{\rho^{2}}{R^{2}}\right)\right] e^{-\rho^{2} / R^{2}}, \\
R^{2}=a^{2}+2 B, \quad \gamma=\frac{\left(1+\epsilon^{2}\right) \sigma_{s}}{8 \pi B}=0.69, \\
\sigma_{s, \text { le }}=2 \int d^{2} \rho\left[\left[1-e^{-\Phi(\rho)} \cos (\epsilon \Phi(\rho))-\frac{1}{2}\left(1-e^{-2 \Phi(\rho)}\right)\right],\right. \\
\sigma_{s, \text { tot }}=2 \int d^{2} \rho\left[1-e^{-\Phi(\rho)} \cos (\epsilon \Phi(\rho))\right], \\
\sigma_{s, \text { dis }}=\int d^{2} \rho\left[e^{-(2-\gamma) \Phi(\rho)}-e^{-2 \Phi(\rho)}\right], \\
\sigma_{s, \text { inel }}=\int d^{2} \rho\left[1-e^{-(2-\gamma) \Phi(\rho)}\right] .
\end{gathered}
$$

The effects of parity violation are a consequence of the interference between the amplitudes of weak and strong interactions. They are linear in the contribution $T_{W}^{p N}$ of weak interactions to the proton-nucleon scattering amplitude, which was considered by us in [23]:

$$
\begin{gathered}
T_{W}^{p p}\left(\mathbf{q}_{\perp}\right)=\lambda_{1} \delta_{\lambda_{1} \lambda_{2}} \delta_{\lambda_{1} \lambda_{3}} \delta_{\lambda_{1} \lambda_{4}} t_{W}^{p p}\left(\mathbf{q}_{\perp}\right), \\
T_{W}^{p n}\left(\mathbf{q}_{\perp}\right)=\lambda_{1} \delta_{\lambda_{1} \lambda_{3}} \delta_{\lambda_{2} \lambda_{4}} t_{W}^{p n}\left(\mathbf{q}_{\perp}\right), \\
t_{W}^{p p}\left(\mathbf{q}_{\perp}\right)=c_{p p} R\left(\mathbf{q}_{\perp}\right), \quad t_{W}^{p n}\left(\mathbf{q}_{\perp}\right)=c_{p n} F^{2}\left(\mathbf{q}_{\perp}\right), \\
F\left(\mathbf{q}_{\perp}\right)=\frac{\Lambda^{4}}{\left(\Lambda^{2}+q_{\perp}^{2}\right)^{2}}, \\
R\left(\mathbf{q}_{\perp}\right)=\frac{4}{\pi} \int \frac{F^{2}\left(\mathbf{k}_{\perp}\right) d^{2} k_{\perp}}{\left(\mathbf{k}_{\perp}-\mathbf{q}_{\perp}\right)^{2}+m_{\rho}^{2}} \\
c_{p p}=5 \mathrm{nb}, \quad c_{p n}=-7.8 \mathrm{nb}, \\
\Lambda=1 \mathrm{GeV}, \quad m_{\rho}=770 \mathrm{MeV} .
\end{gathered}
$$

The corresponding contribution $\mathscr{T}_{W}(\boldsymbol{\rho})$ to the function $\mathscr{T}(\boldsymbol{\rho})$ in Eq. (1) is purely imaginary, $\mathscr{T}_{W}(\rho)=i \xi(\rho)$, where

$$
\begin{gathered}
\xi(\rho)=\frac{1}{2} \int \frac{d^{2} Q_{\perp}}{(2 \pi)^{2}} e^{i \mathbf{Q}_{\perp} \cdot \rho} \\
\times\left[\frac{1}{2} t_{W}^{p p}\left(\mathbf{Q}_{\perp}\right)+t_{W}^{p n}\left(\mathbf{Q}_{\perp}\right)\right] S\left(\mathbf{Q}_{\perp}\right) .
\end{gathered}
$$

Here, the factor $1 / 2$ in front of $t_{W}^{p p}\left(\mathbf{Q}_{\perp}\right)$ appears because the amplitude $T_{W}^{p p}$ of the weak interaction is nonzero only for protons of the nucleus with the same helicity as that of the incident proton $\left(\lambda_{2}=\lambda_{1}\right)$.

The correction $\Omega_{W}(\boldsymbol{\rho})$ to the function $\Omega(\rho)$ in Eq. (4) due to weak interactions has the form

$$
\begin{gathered}
\Omega_{W}(\boldsymbol{\rho})=-\frac{1}{2} \epsilon \sigma_{s} \\
\times \iint \frac{d^{2} Q_{\perp}}{(2 \pi)^{2}} \frac{d^{2} Q_{\perp}^{\prime}}{(2 \pi)^{2}} e^{i\left(Q_{\perp}-Q_{\perp}^{\prime}\right) \cdot \boldsymbol{\rho}} S\left(Q_{\perp}^{\prime}-Q_{\perp}\right) \\
\times\left[\frac{1}{2} t_{W}^{p p}\left(\mathbf{Q}_{\perp}^{\prime}\right)+t_{W}^{p n}\left(\mathbf{Q}_{\perp}^{\prime}\right)\right] \exp \left(-\frac{1}{2} B Q_{\perp}^{2}\right) .
\end{gathered}
$$

As a result, we find the corrections to the cross sections due to weak interactions:

$$
\begin{gathered}
\sigma_{W, \mathrm{el}}=\sigma_{W, \text { tot }}-2 \int d^{2} \rho e^{-\Phi(\rho)} \sin (\epsilon \Phi(\rho)) \xi(\rho), \\
\sigma_{W, \text { dis }}=-\sigma_{W, \text { inel }}=\int d^{2} \rho e^{-(2-\gamma) \Phi(\rho)} \Omega_{W}(\rho) .
\end{gathered}
$$

\section{DISCUSSION OF THE RESULTS}

We pass now to numerical estimates of the cross sections and the corresponding asymmetries $\mathscr{A}=\sigma_{W} / \sigma_{s}$ for the scattering of a polarized proton 
with $\lambda_{1}=1$ by carbon and oxygen nuclei. Using the above formulas, we find for ${ }^{12} \mathrm{C}$ :

$$
\begin{aligned}
& \sigma_{s, \text { tot }}^{p \mathrm{C}}= 410 \mathrm{mb}, \quad \sigma_{W, \text { tot }}^{p \mathrm{C}}=-3.7 \mathrm{nb}, \\
& \mathscr{A}_{\mathrm{tot}}^{p \mathrm{C}}=-0.9 \times 10^{-8}, \\
& \sigma_{s, \mathrm{el}}^{p \mathrm{C}}= 126 \mathrm{mb}, \quad \sigma_{W, \mathrm{el}}^{p \mathrm{C}}=-3.7 \mathrm{nb}, \\
& \mathscr{A}_{\mathrm{el}}^{p \mathrm{C}}=-2.9 \times 10^{-8} \\
& \sigma_{s, \text { inel }}^{p \mathrm{C}}= 228 \mathrm{mb}, \quad \sigma_{W, \text { inel }}^{p \mathrm{C}}=1 \mathrm{nb}, \\
& \mathscr{A}_{\text {inel }}^{p \mathrm{C}}=4.4 \times 10^{-9} \\
& \sigma_{s, \mathrm{dis}}^{p \mathrm{C}}=56 \mathrm{mb}, \quad \sigma_{W, \mathrm{dis}}^{p \mathrm{C}}=-1 \mathrm{nb}, \\
& \mathscr{A}_{\mathrm{dis}}^{p C}=-1.8 \times 10^{-8} .
\end{aligned}
$$

For ${ }^{16} \mathrm{O}$, we obtain:

$$
\begin{aligned}
\sigma_{s, \text { tot }}^{p \mathrm{O}}= & 517 \mathrm{mb}, \quad \sigma_{W, \text { tot }}^{p \mathrm{O}}=-4.8 \mathrm{nb}, \\
& \mathscr{A}_{\text {tot }}^{p \mathrm{O}}=-0.9 \times 10^{-8}, \\
\sigma_{s, \mathrm{el}}^{p \mathrm{O}}= & 169 \mathrm{mb}, \quad \sigma_{W, \mathrm{el}}^{p \mathrm{O}}=-4.8 \mathrm{nb}, \\
& \mathscr{A}_{\mathrm{el}}^{p \mathrm{O}}=-2.7 \times 10^{-8}, \\
\sigma_{s, \text { inel }}^{p \mathrm{O}}= & 284 \mathrm{mb}, \quad \sigma_{W, \text { inel }}^{p \mathrm{O}}=1.2 \mathrm{nb}, \\
& \mathscr{A}_{\text {inel }}^{p \mathrm{O}}=4.2 \times 10^{-9}, \\
\sigma_{s, \mathrm{dis}}^{p \mathrm{O}}= & 64 \mathrm{mb}, \quad \sigma_{W, \text { dis }}^{p \mathrm{O}}=-1.2 \mathrm{nb}, \\
& \mathscr{A}_{\mathrm{dis}}^{p \mathrm{O}}=-1.8 \times 10^{-8} .
\end{aligned}
$$

It is seen that the asymmetry is most noticeable in the elastic cross section and in the dissociation cross section. As we noted in $[23,24]$ when analyzing nucleonnucleon and nucleon-deuteron scattering, the suppression of asymmetry in inelastic scattering is a consequence of the unitarity condition. Therefore, from the experimental point of view, it seems advantageous to measure the asymmetry either in elastic scattering or in the sum of the elastic cross section and the dissociation cross section, for which

$$
\mathscr{A}_{\mathrm{el}+\mathrm{dis}}^{p \mathrm{C}} \approx \mathscr{A}_{\mathrm{el+ \textrm {dis }}}^{p \mathrm{O}}=-2.6 \times 10^{-8} .
$$

The general conclusion from the comparison of $p \mathrm{C}$ and $p \mathrm{O}$ scattering with $p N$ and $p d$ scattering studied in $[23,24]$ is the following. The expected $P$-odd asymmetries in the $p \mathrm{C}$ and $p \mathrm{O}$ scattering cross sections are very close to each other and differ by less than $10 \%$ from the analogous values in the scattering of protons by unpolarized deuterons. This is due to the fact that the contributions of the strong and weak interactions to the cross sections for the scattering of protons by nuclei grow approximately the same with an increase in the number of nucleons in the nucleus. An exception is the quasi-elastic scattering, in which we predict an increase in asymmetry in $p \mathrm{C}$ and $p \mathrm{O}$ scattering by almost two times compared to $p d$ scattering. As in the case of a deuteron target, the proton and neutron cor- rections to the cross sections due to the weak interaction partially compensate each other, so that in scattering by nuclei the $P$-odd asymmetry is less than in $p p$ scattering. However, from an experimental point of view, both proton (hydrogen) and deuterium targets can be less convenient due to their low densities.

\section{CONCLUSIONS}

We have analyzed the effects of parity violation in the scattering of polarized protons by the nuclei ${ }^{12} \mathrm{C}$ and ${ }^{16} \mathrm{O}$ at energies of the NICA collider. Using the Glauber approach, we obtained estimates for the weak interaction corrections to the total, elastic, inelastic and dissociation cross sections, as well as the corresponding spin asymmetries, see (11) and (12). According to our results, experiments on measuring the asymmetry in the cross section $\sigma_{\mathrm{el}}$ or $\sigma_{\mathrm{el}+\mathrm{dis}}$ are preferable. The results obtained should be taken into account when planning experiments at the NICA collider.

\section{FUNDING}

This work was supported by the Russian Foundation for Basic Research (project no. 18-02-40092 MEGA).

\section{CONFLICT OF INTEREST}

The authors declare that they have no conflicts of interest.

\section{OPEN ACCESS}

This article is licensed under a Creative Commons Attribution 4.0 International License, which permits use, sharing, adaptation, distribution and reproduction in any medium or format, as long as you give appropriate credit to the original author(s) and the source, provide a link to the Creative Commons license, and indicate if changes were made. The images or other third party material in this article are included in the article's Creative Commons license, unless indicated otherwise in a credit line to the material. If material is not included in the article's Creative Commons license and your intended use is not permitted by statutory regulation or exceeds the permitted use, you will need to obtain permission directly from the copyright holder. To view a copy of this license, visit http://creativecommons.org/licenses/by/4.0/.

\section{REFERENCES}

1. J. M. Potter, J. D. Bowman, C. F. Hwang, J. L. McKibben, R. E. Mischke, D. E. Nagle, P. G. Debrunner, H. Frauenfelder, and L. B. Sorensen, Phys. Rev. Lett. 33, 1307 (1974).

2. D. E. Nagle, J. D. Bowman, C. Hoffman, J. McKibben, R. Mischke, J. M. Potter, H. Frauenfelder, and L. Sorenson, AIP Conf. Proc. 51, 224 (1978). 
3. R. Balzer, R. Henneck, Ch. Jacquemart, J. Lang, M. Simonius, W. Haeberli, Ch. Weddigen, W. Reichart, and S. Jaccard, Phys. Rev. Lett. 44, 699 (1980).

4. N. Lockyer, T. A. Romanowski, J. D. Bowman, C. M. Hoffman, R. E. Mischke, D. E. Nagle, J. M. Potter, R. L. Talaga, E. C. Swallow, D. M. Alde, D. R. Moffett, and J. Zyskind, Phys. Rev. D 30, 860 (1984).

5. V. Yuan, H. Frauenfelder, R. W. Harper, J. D. Bowman, R. Carlini, D. W. MacArthur, R. E. Mischke, D. E. Nagle, R. L. Talaga, and A. B. McDonald, Phys. Rev. Lett. 57, 1680 (1986).

6. P. D. Eversheim, W. Schmitt, S. Kuhn, F. Hinterberger, P. von Rossen, J. Chlebek, R. Gebel, U. Lahr, B. von Przeworski, M. Wiemer, and V. Zell, Phys. Lett. B 256, 11 (1991).

7. A. R. Berdoz, J. Birchall, J. B. Bland, et al., Phys. Rev. C 68, 034004 (2003).

8. V. Brown, E. Henley, and F. Krejs, Phys. Rev. C 9, 935 (1974).

9. E. M. Henley and F. R. Krejs, Phys. Rev. D 11, 605 (1975).

10. V. B. Kopeliovich and L. L. Frankfurt, JETP Lett. 22, 295 (1975).

11. L. L. Frankfurt and V. B. Kopeliovich, Nucl. Phys. B 103, 360 (1976).

12. B. Desplanques, J. Donoghue, and B. Holstein, Ann. Phys. (N. Y.) 124, 449 (1980).

13. L. L. Frankfurt and M. I. Strikman, Phys. Lett. B 107, 99 (1981).

14. A. Barroso and D. Tadić, Nucl. Phys. A 364, 194 (1981).

15. T. Oka, Prog. Theor. Phys. 66, 977 (1981).
16. G. Nardulli and G. Preparata, Phys. Lett. B 117, 445 (1982).

17. T. Goldman and D. Preston, Nucl. Phys. B 217, 61 (1983).

18. B. G. Zakharov, Sov. J. Nucl. Phys. 42, 479 (1985).

19. V. D. Kekelidze, R. Lednicky, A. Matveev, I. N. Meshkov, A. S. Sorin, and G. V. Trubnikov, in Proceedings of the 3rd Large Hadron Collider Physics Conference LHCP 2015 (2016), p. 565.

20. I. Savin, A. Efremov, D. Peshekhonov, A. Kovalenko, O. Teryaeva, O. Shevchenko, A. Nagajcev, A. Guskov, V. Kukhtin, and N. Toplilin, EPJ Web Conf. 85, 02039 (2015).

21. I. A. Koop, A. I. Milstein, N. N. Nikolaev, A. S. Popov, S. G. Salnikov, P. Yu. Shatunov, and Yu. M. Shatunov, Phys. Part. Nucl. Lett. 17, 154 (2020).

22. I. A. Koop, A. I. Milstein, N. N. Nikolaev, A. S. Popov, S. G. Salnikov, P. Yu. Shatunov, and Yu. M. Shatunov, Phys. Part. Nucl. 52, 549 (2021).

23. A. I. Milstein, N. N. Nikolaev, and S. G. Salnikov, JETP Lett. 111, 197 (2020).

24. A. I. Milstein, N. N. Nikolaev, and S. G. Salnikov, JETP Lett. 112, 332 (2020).

25. R. J. Glauber, Phys. Rev. 100, 242 (1955).

26. V. Franco and R. J. Glauber, Phys. Rev. 142, 1195 (1966).

27. R. J. Glauber and G. Matthiae, Nucl. Phys. B 21, 135 (1970).

28. J. Ryckebusch, D. Debruyne, P. Lava, S. Janssen, B. van Overmeire, and T. van Cauteren, Nucl. Phys. A 728, 226 (2003). 